\title{
Über die Beziehungen zwischen Genitiv und Possessivadjektiv im Tscheremissischen
}

Nach der herkömmlichen Auffassung war der in den finnischwolgaischen Sprachen vorkommende Genitiv ursprünglich eine possessive Adjektivableitung. Speziell im Tscheremissischen soll die ursprüngliche Funktion immer noch spürbar sein. Mit anderen Worten wird vorausgesetzt, dass die Genitivendung in einigen Fällen gar nicht als Kasussuffix zu deuten ist, sondern als Adjektivableitungssilbe.

Das tscheremissische Genitivsuffix begegnet in zwei morphophonetischen Varianten: $n$ und $ә n$, der Lautwert des reduzierten Vokals schwankt dialektal nach der Umgebung. Jedenfalls steht ein blosses $n$ nach einem auf einen Vokal auslautenden Wortstamm, reduzierter Vokal $+n$ nach einem auf einen Konsonanten endenden Wortstamm: šińča 'Auge' : šincéa-n 'des Auges', bata 'Frau' : batə-n 'der Frau', surt 'Haus' : surt-ən 'des Hauses'.

Einige Formen, die wie Genitive aussehen, sind jedoch nicht eindeutig. Im heutigen Tscheremissisch gibt es auch ein possessives Adjektivableitungssuffix, das ebenfalls in Form von zwei Allomorphen auftritt: $n$ und an (in Dialekten mit $\ddot{a}$-Harmonie kommt bei vordervokalischen Wortstämmen auch än in Frage). Das blosse $n$ tritt an die auf $a(\ddot{a})$ endenden Wortstämme, an(än) an alle anderen Stammtypen: šinčca-n 'äugig' von šińča, bat-an 'verheirateter Mann' von batz, surt-an 'Hausbesitzer' von surt. Die Genitivendung und das Adjektivableitungssuffix erzeugen also bei den auf $a(\ddot{a})$ endenden Wörtern homonyme Formen: šińčan ist auf zwei Arten zu deuten, entwerder šinča $\{\mathrm{N}\}$ oder šińč $\{\mathrm{AN}\}$, um morphophonemische Symbole zu verwenden.

Hinsichtlich ihrer morphologischen Distribution unter- 
scheiden sich jedoch die Genitivendung und das Suffix des Possessivadjektivs deutlich voneinander. Wenn man den adnominal verwendeten Genitiv und den adverbal verwendeten Instruktiv nicht als verschiedene Formkategorien betrachtet - eine Auffassung, die Wiklund, Ravila und E. Itkonen vertreten - , hat das Auftreten des Genitivsuffixes keinerlei morphologische Begrenzungen. Der Genitivfall kann von jedem beliebigen Nominalstamm und auch von jedem beliebigen Verbalstamm gebildet werden; das von Wichmann erwähnte sog. Verbalnomen V konden 'tragend, getragen habend', piðən 'bindend, gebunden habend' ist formal ja ein klarer Genitiv. Das Suffix des Possessivadjektivs tritt dagegen nicht an den Verbalstamm mit Ausnahme solcher Sonderfälle wie ulanžam, ukanžam oyeš šińd'ž́e 'er weiss nicht was er hat und was er nicht hat' (Wb 421) ${ }^{1}$, uke tritt ja auch als deutliches Nomen auf und zieht in Parallelausdrücken den Stamm ulo mit sich. Das ANSuffix verbindet sich ausserdem auch nicht mit jedem beliebigen Nominalstamm. Vom Wort lele lela 'schwer' z.B. begegnet der Genitiv lelan 'schwer (Adv.)', ein Possessivadjektiv lässt sich daraus jedoch nicht bilden. Dies ist übrigens die einzige morphologische Grundlage, auf der sich im Tscheremissischen die Substantive und die Adjektive in verschiedene Kategorien einteilen lassen: die Wörter, an die das ANSuffix treten kann, sind Substantive, die anderen sind Adjektive. Unberücksichtigt bleiben bei dieser Einteilung die Pronomina und die Zahlwörter, von denen keine Possessivadjektive gebildet werden können, die syntaktisch aber zu beiden Gruppen gehören können. Mit anderen Worten werden Adjektive nicht von Wörtern gebildet, die ohnehin Adjektive sind. An die Genitivform kann das AN-suffix nicht angefügt werden, obgleich es auch Ausnahmen von dieser Regel gibt: lat-kok šör-ən-an lupšəm pualna 'wir geben eine zwölfgeteilte Peitsche' Bu2 465 und aus dem Wörterbuch von Serebrennikov: kum vož-an-an šańək 'dreizinkige Mistgabel'. In diesen Fällen vertritt die Genitivform zwar den Wortstamm

1 Die Übersetzung der Textquellen wurde verändert, wo dies zwecks Verdeutlichung für nötig befunden wurde. 
und das Genitivsuffix erscheint als Ableitungselement. Ein auf diese Weise gebildetes Wort ist jedoch kein Adjektiv sondern ein Substantiv, wie oben nachgewiesen wurde. Beachtenswert ist, dass ein possessives Adjektiv den vorliegenden Quellen nach nie in der Genitivform erscheint, von diesen Ableitungen lassen sich also offenbar keine adverbalen Genitive bilden, wie von allen anderen Adjektiven. So tritt das N-Suffix an alle Substantive, alle Verben und alle Adjektive mit Ausnahme der Possessivableitungen, das AN-Suffix dagegen wird an alle Substantive angehängt.

Der eigentliche Vergleich zwischen Genitiv und Possessivadjektiv muss natürlich auf syntaktischer Grundlage unter Berücksichtigung sowohl der adnominalen als auch der adverbalen Funktionen des Genitivs vollzogen werden. Die syntaktische Funktion einer AN-Ableitung ist die eines Adjektivs überhaupt: im Satz als direkte Bestimmung, als Attribut, neben einem Nomen zu stehen oder in einem Nominalsatz Prädikativum zu sein: izi üdər 'kleines Mädchen', üdər izi 'das Mädchen ist klein', kuž-üpan üdər 'langhaariges Mädchen', üdər kuž-üpan 'das Mädchen ist langhaarig'. In diesen Stellungen ist das Adjektiv unflektierbar, mit Ausnahme einiger Kongruenzfälle, wo ein Adjektivattribut, das zu einem im Akkusativ stehenden Beziehungswort tritt, die Endung dieses Beziehungswortes erhält. Die Deklination ist für die Adjektive nicht charakteristisch, die einzelnen Kasusformen der Adjektive sind erstarrte, substantivierte Ausdrücke. Der einzige oblique Kasus der Adjektive ist gerade der Genitiv, doch kommt er, wie erwähnt, bei den Possessivadjektiven nicht vor.

Die syntaktischen Funktionen des Genitivs sind recht vielfältig. Der adnominale Genitiv verbindet sich entweder direkt oder durch Vermittlung eines Verbs mit seinem Beziehungswort: jenan pörtšö 'das Haus des Mannes', jenan ulo pörť̌ö 'der Mann hat ein Haus'; zu beachten ist, dass in beiden Fällen im Beziehungswort meist ein auf die Bestimmung hinweisendes Personal- bzw. Possessivsuffix enthalten ist. Der Genitiv kann im Satz auch als adverbale Bestimmung stehen Wichmann nennt dies Instruktiv, z.B. imńan kaja 'fährt mit dem Pferd'; die Verwendung dieser Kasusform ist sehr mannig- 
faltig. Alle Genitive eines Verbalstammes sind hinsichtlich ihrer syntaktischen Verwendung adverbal. Das gilt auch für die Genitive der Adjektive. Bei den letztgenannten könnte das N-Suffix als Adverbableitung betrachtet werden. Der adverbale Nomengenitiv gliedert sich jedoch immer noch dem Kasussystem ein. Er kann ein Adjektivattribut erhalten: maśka toleš, muraš tünaleš kuүo jükən 'der Bär kommt, beginnt mit lauter Stimme zu singen' Gp 24. In ihrer syntaktischen Distribution sind alle drei Typen jedoch völlig identisch: imńan kaja 'geht mit dem Pferd', pisən kaja 'geht schnell', kuržan kaja 'geht laufend'.

Zwischen den beiden Kasusformen der Adjektive herrscht eine deutliche syntaktische Aufteilung: der Nominativ ist adnominal, der Genitiv adverbal. Aufschlussreich ist es, im folgenden Satz die Beziehungen zwischen dem Adjunkt und dem Beziehungswort miteinander zu vergleichen: $\beta$ ijye užat: poro iləšəmat, jösən ilməmat 'alles siehst du, gutes Leben und schweres Leben' Pab 21; iłă̌ 'Leben', ein deverbales Nomen, erhält ein im Nominativ stehendes Adjektivattribut als Bestimmung, il(a)me aber, als Verbalnomen, erhält ein adverbales, im Genitiv stehendes Adjektiv.

Auch der adverbale Nomengenitiv fügt sich reibungslos dem System ein. Dasselbe Verhältnis wie zwischen dem Nominativ und Genitiv eines Adjektivs herrscht auch zwischen einem von einem Nomen abgeleiteten Possessivadjektiv und dem Genitiv eines Nomens: ersteres ist adnominal, letzteres adverbal. Dies wird in Verbindungen deutlich, wo beide nebeneinander, von den gleichen Wörtern gebraucht, auftreten:

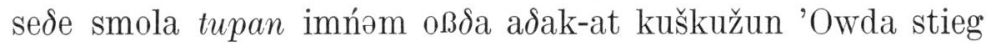
wieder auf das Pferd mit dem mit Pech beschmierten Rücken' Bb1 40.

čəla türlə osaləm tupən poktələn nalən kai 'alle verschiedenen bösen (Sachen) fahr rückwärts fahrend weg' Pt 19. kuyo jükan toleš kəń, i $\delta$ a purto 'wenn der Lautstimmige kommt, lasst ihn nicht hinein'.

maśka toleš, muraš tünaleš kưo jükan 'der Bär kommt, beginnt mit lauter Stimme zu singen'.

ta $\delta$ e ku $\gamma_{0}$ jükan 'das ist der Lautstimmige'. Alle Beispiele stammen aus der Quelle Gp, Seite 24. 
kinDe-kaßan mṇskṇrṇn lekteš tek, tənam ßate mṇsknnran liješ 'wird der Getreideschober bauchig, so wird das Weib schwanger' Bja 63. šem imńan il’tťs, oš imńanßlak ertəšt 'sie waren Schwarzpferdige (sie hatten schwarze Pferde), die Weisspferdigen fuhren vorüber' $\mathrm{Pab} 17$.

kaja korno Bokten jolan imńin '[er] reitet seines Weges' ("menee jalkahevosella») $\mathrm{Bj}^{\mathrm{b}} 317$.

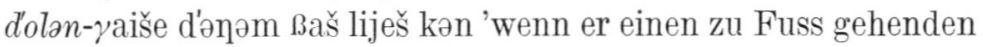
Mann trifft' Bt 542.

porśən melan tußurem 'mein seidenbrüstiges Hemd' Bm 120. Patambal arka kečəlan melən 'der oberhalb des Patam-Stromes liegende Abhang liegt gegen die Sonne' Gp 73.

Das Possessivadjektiv und der adverbale Genitiv treten also ihrem syntaktischen Gebrauch nach als Gegenpole auf. Der adnominale bzw. possessive Genitiv behält dagegen eine gewisse Sonderstellung. Er vertritt eine verhältnismässig seltene syntaktische Kategorie. Wie ich in meiner Dissertation erwähnte, wird er vor allem bei Wörtern verwendet, die belebte Begriffe bezeichnen. Bezeichnend ist, dass in Gedichten "unbelebte» Genitive verhältnismässig öfter vorkommen als in der Prosa, offenbar eine Folge der Personifikation. Die Anzahl der unbelebten adnominalen Genitive bleibt in den meisten Textquellen unter $3 \%$, in der umfangreichen Sammlung von Beke aus Uržum (MSFOu 76), die nur Prosa enthält, sogar unter $1 \%$. Der allgemeine Kasus des adnominalen Adjunkts ist der Nominativ, bei unbelebten Nomina fast ausschliesslich. Beispiele gibt es sehr viel: imńan bujžo 'Kopf des Pferdes', aber korno buj 'Ende des Weges' und auch imńe buj oder imńe bujžo, da es keine Beschränkungen gibt für das Auftreten eines im Nominativ stehenden Adjunkts.

Der Possessivgenetiv und das Possessivadjektiv treten beide als adnominale Bestimmungen auf, so dass ihre syntaktischen Funktionen zusammenzufallen scheinen. Ein grundsätzlicher Unterschied kommt jedoch bereits darin zum Ausdruck, dass das AN-suffix zur Bildung von Adjektiven ebenso gut an Wortstämme tritt, die belebte wie unbelebte Begriffe bezeichnen: sem imńan ilotšs 'sie hatten schwarze Pferde', eig. 'sie waren Schwarzpferdige' (Pab), kornan tubər 'gestreiftes Hemd' (Jewsky). 
Syntaktisch gesehen zeigt sich der Unterschied darin, dass der adnominale Genitiv als Genitivattribut auftritt, das Possessivadjektiv — wie die Adjektive im allgemeinen — als Adjektivattribut. Obgleich beide die unmittelbaren Bestimmungen des Nomens sind, lassen sie sich nicht ganz gleichstellen. Dies merkt man am besten an Fällen, wo das Nomen gleichzeitig sowohl ein Genitiv- als auch ein Adjektivattribut besitzt: jenan poro imńaže. Der Ausdruck bildet eine Gesamtheit, deren syntaktische Funktion von der Form des Beziehungswortes abhängt, imńaže also ist das Kernglied des Ausdrucks. Mit dem Beziehungswort sind zwei Adjunkte verbunden, jedoch nicht gleichwertig; p o r o i mń ә ̌̌ e bildet vielmehr ein Ganzes, das von jenan bestimmt wird, also 'des Mannes gutes Pferd'. Wenn die Adjunkte ihre Plätze wechseln: poro jenan imńaže, ändert sich auch die syntaktische Struktur des Ausdrucks und gleichzeitig seine Bedeutung: nun bilden poro und jenan eine Gesamtheit, die das Wort i mń əž e bestimmt: 'des guten Mannes Pferd'. (Als Adjektivattribut kann natürlich auch ein Possessivadjektiv stehen: jenən š i j-tag a n a n imń ə ž e 'des Mannes silberhufiges Pferd' oder šij-taganan imńan o z a ž e 'des silberhufigen Pferdes Herr'.) Das Adjunkt in Genitivform vermag den Zusammenhang zwischen Adjektiv und Beziehungswort zu durchbrechen. Das Adjektivattribut ist für das Nomen also eine Art Bestimmung ersten Grades, das Genitivattribut nur eine Bestimmung zweiten Grades. Zwischen dem Adjektivattribut und seinem Beziehungswort wie auch zwischen dem Subjekt und dem als Prädikativum stehenden Adjektiv in Nominativform in einem Nominalsatz herrscht eine vollständige begriffliche Entsprechung; sie decken einander ganz: kugu pört 'grosses Haus', pört kugu 'das Haus ist gross'. Das Genitivadjunkt und sein Beziehungswort decken sich dagegen i.a. begrifflich nur teilweise oder gar nicht: jenan pörť̌ö 'des Mannes Haus', jenan buǰ̆o 'des Mannes Kopf'. Es gibt auch Beispiele für eine vollständige Entsprechung: jenən porəžo 'des Mannes Güte', doch ist zu beachten, dass ein solcher Ausdruck nicht in einen Nominalsatz zerlegt werden kann; *porəž jẹan ist begrifflich nicht möglich.

Eine Sondergruppe der Genitivbelege bilden die Fälle, wo 
der Genitiv im Satz selbständig geworden ist und ohne ein durch das Genitivsuffix vorausgesetztes Beziehungswort steht. Der Genitiv ist dann mit dem Wortstamm vergleichbar und das Genitivsuffix hat als Ableitungssuffix zu gelten, an das

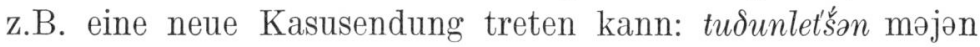
əškemən saj 'meins ist besser als seins' Bb2 392. Anzumerken ist, dass die Genitive auch im Finnischen im entsprechenden Ausdruck 'minun on parempi kuin hänen' an sich als Ableitungen auftreten, ohne Beziehungswort, obgleich sie im Bedarfsfall keine Kasusendungen erhalten können. Im Finnischen pflegt man solche Genitive als elliptisch zu bezeichnen und als Attribute zu gliedern. Den Belegen liegt jedoch die Tatsache zugrunde, dass die Bestimmung mit dem Wegfall des Beziehungswortes die syntaktische Funktion des letzteren erbt. Im Tscheremissischen ist die Verselbständigung des Genitivs gewöhnlicher und klarer als im Finnischen, vgl. die Beispiele von Jewsky: šlapažəm nalneže, naleš tudanəm (Gen. + Akk.) '(der Mann) will den Hut nehmen, er nimmt seinen (d.h. den Hut eines anderen)'. Mitunter erscheint der selbständige Genitiv in Stellungen, wo kein ergänzendes Nomen stehen kann. Setälä nennt in seiner Gemeinfinnischen Lautgeschichte im Zusammenhang mit der Adjektivität des tscheremissischen Genitivs gerade einen solchen Fall: śkendanəm nal, jęənəm mönge pu 'nimm dein eigenes, gib zurück was den andern gehört (eig. der anderen ist)'. Wenn man hier auch von einer Possessivableitung sprechen könnte, handelt es sich doch um ein possessives Substantiv, kein Adjektiv. Ausserdem lässt sich die Erscheinung aufgrund des adnominalen Genitivs, aufgrund der Verselbständigung des Genitivs erklären. Derartige Fälle bleiben somit ausserhalb unserer Untersuchung.

Falls die Annahme belegt werden soll, wonach der Genitiv im Tscheremissischen als Possessivadjektiv erscheint, müssen die Fälle aufgezählt werden, wo ein im Genitiv stehendes Nomen in derselben syntaktischen Stellung auftritt wie ein Adjektiv in Grundform: als erstgradige Bestimmung des Nomens also oder als Prädikativum im Nominalsatz.

Ich habe das in meiner Dissertation genannte Textmaterial durchgesehen und alle in Frage kommenden Beispiele gesam- 
melt. Zu beachten ist dabei, dass das eventuelle Belegmaterial der auf $a, \ddot{a}$ auslautenden Wörter nicht berücksichtigt werden kann. Wie im Anfang erwähnt, sind der Genitiv und die possessive Adjektivableitung bei diesen homonym, so dass man nie sagen kann, um welche Form es sich handelt. Lewy nennt in seiner Grammatik als Beispiel für die Adjektivität des Genitivs im Tscheremissischen onže pel-šinčan 'der Onkel ist einäugig', das somit keine Beweiskraft hat. Im Gegenteil weisen die Parallelbeispiele von Wörtern, deren Genitiv und Possessivadjektiv verschiedene Formen haben, darauf hin, dass das pel-šinčan in Lewys Beispiel eher als Possessivadjektiv zu deuten wäre: k'latem pel-jolan 'mein Speicher hat einen Fuss' (Gp 48; vom Wort jol lautet der Genitiv jolan und das Possessivadjektiv jolan, während šinčan formal sowohl ein Genitiv als auch ein Possessivadjektiv sein kann). Aus der Schriftsprache stammt das Beispiel müden šəndəmə litsaan 'Honig-befleckt-Gesichtiger' I 6, wo man mit Hilfe der Schreibweise hat zeigen wollen, dass es sich wirklich um eine Adjektivableitung handelt und nicht um einen Genitiv.

Auch die Wörterbuchbelege sind nicht geeignet, den Ausgangspunkt für die Untersuchung zu bilden, einzelne Genitivformen, die in ihrer Bedeutung zwar zur Erläuterung der Sachlage beizutragen scheinen. In der Wortzettelsammlung von Wichmann ist beim Stichwort ij 'Eis' eine Reihe von Genitivformen aufgezählt: in 'aus Eis, eisig', tuman 'aus Eiche', kožan 'aus Fichte', jäktan 'aus Kiefer', pun 'hölzern', kartniin 'eisern', lun 'knöchern', alle aus dem Dialekt von Kozmodemjansk. Ein Hinweis auf die syntaktische Distribution wird nur für den im gleichen Zusammenhang erwähnten Typus iän gegeben: iän Büt 'eisvermischtes Wasser'. Es wäre sehr wichtig zu erfahren, ob in und iän in genau gleichen syntaktischen Stellungen vorkommen.

Die Beispiele finden sich gleichmässig in allen Dialekten. Es kommen aber nur relativ wenige Wörter in Frage. Im ganzen habe ich in meinem Textmaterial 17 als Adjektivattribute zu deutende Genitivformen eines Nomens gefunden und 36 Belege für ein Prädikativum. Ausserdem erscheint der Genitiv in vier 
Fällen substantiviert. Beim überwiegenden Teil der Belege handelt es sich um Varianten eines Sprichworts oder Rätsels in den einzelnen Dialekten. In einigen Fällen beruht die Genitivform auf einer Art Systemzwang, z.B. durch den Einfluss eines im Parallelsatz stehenden possessiven Genitivs.

Im folgenden zähle ich die in meinen Textquellen als Adjektive auftretenden Genitivformen auf, wobei ich die Häufigkeit der Belege sowie die Quellen verzeichne.

A. Die Genitivform eines Nomens erscheint als Adjektivattribut.

božan vom Wort bož 'Zweig' : ßerešteš $\beta$ žzวn kornə 'sie kommen an eine Weggabelung (eig. 'es kommt ein Zweigweg') Bu1 263. Die Form begegnet als erstes Glied eines aus einem Kompositum gebildeten Adjektivs in dem Ausdruck božanpočan: $\beta_{0}$ žn-počan Baraksimžəm Barsitlaš koldəšna 'wir schickten eine Schwalbe mit verzweigtem Schwanz zu zwitschern' Pt 54. In verschiedenen Quellen insgesamt 5 Beispiele.

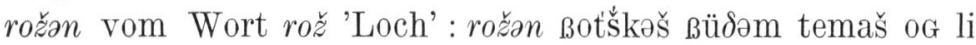
'es lohnt sich nicht, Wasser in ein löchriges Fass zu schöpfen' Wt 129. Insgesamt 7 Beispiele, 5 in den Sprichwörtervarianten der Texte von Jevsevjev, 1 im Werk Elnet von Chavain. puən vom Wort pu 'Baum, Holz' : ki əəštem ulo šište kuðər korka, Onatər puən korkaže 'in meiner Hand ist ein maserhölzerner Becher, ein aus Onatər-Holz gemachter Becher' Gp 74.

tumən vom Wort tumo 'Eiche' : Bara mare-šaməts tsəla jambəlen olt, pikšəm, kestenəm tumənəm 'danach haben die Tscheremissen alles gerüstet, die Pfeile, die Eichenkeulen' Bjc 182. Es handelt sich hier zwar um einen appositionsartigen, verselbständigten Genitiv.

šöran vom Wort šör 'Kante' : pasu pokšelnə šörən kußa košteš 'mitten auf der Wiese geht eine krumme Frau' Pt 28. Ein zweites Beispiel findet sich in demselben Rätsel in der Quelle Bu2.

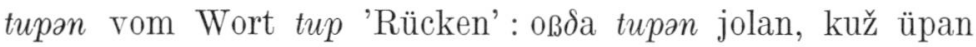
'Owda hat verkehrte Beine, langes Haar' Bu2 465. 
B. Die Genitivform eines Nomens erscheint als adjektivisches Prädikativum.

božวn: nemnan kornəna vožวn 'unser Weg ist verzweigt, d.h. unsere Wege haben sich getrennt' M 45. Insgesamt 8 Beispiele.

rožan: kutanže rožan, nerže $\beta$ ožan 'sein Hinterteil ist löchrig, seine Nase ist gespalten' Gp 45. - Für die als Prädikativum auftretende Form rožan liefern die verschiedenen Quellen 10 Beispiele.

pun: Saßəli äpšätən, Apati pun 'Sabellius gehört dem Schmied, Apati ist aus Holz' Wkb 135. 2 Beispiele, dasselbe Rätsel. kobaštan vom Wort kobašte 'Leder';

šörtnian vom Wort šörtńö 'Gold' : iktəže šörtńan, maneš, Besəž koßaštan liješ 'der eine ist aus Gold, sagt er, der andere aus Leder' $\mathrm{Bj}^{\mathrm{b}}$ 161. Die erstere Form begegnet in den Textquellen einmal, die letztere zweimal.

knnrtńnnn vom Wort knnrtńn 'Eisen' : bedra il’ kürtńün 'der Eimer war aus Eisen' Lt 37. Insgesamt 3 Beispiele.

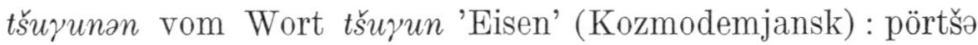

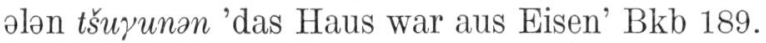

kuen vom Wort kue 'Birke', kožzn vom Wort kož 'Fichte': Bijakš kuen paškarže, Bijakš kožən onaže 'aus gerader Birke verfertigter Geigenwirbel, aus gerader Fichte verfertigtes Brett' Bjb 360. Für die Form šorakan gibt es zwei, für kuen 3 Belege in den Varianten desselben Liedes. In der Quelle Bt treten noch einige andere Bäume auf: tuman, šolan vom Wort šolo 'Weide', piśtan vom Wort piśte 'Linde': jolžo tuman, piðəšəže šolan, öretšaže piśtan 'seine Streben sind aus Eiche, seine Bindungen aus Weide, seine »Flügel» aus Linde’ Bt 387.

C. Die Genitivform tritt als substantiviertes Adjektiv auf.

šörpnan: ni-manat kote šördrian 'es blieb nichts was aus Gold war' Bkb 242.

vožวn: tumo vožanam šeleš 'er spaltet den Ast der Eiche' Ch 17. šöran: luatkok šörnan lupšəm jodeš 'er bittet um die zwölfgeteilte Peitsche' Jt F I Nr. 305. Insgesamt zwei Beispiele, in beiden ist die Genitivform das Stammwort für das Possessivadjektiv. 
In der folgenden Tabelle sind alle adjektivischen Belege des Genitivs verzeichnet.

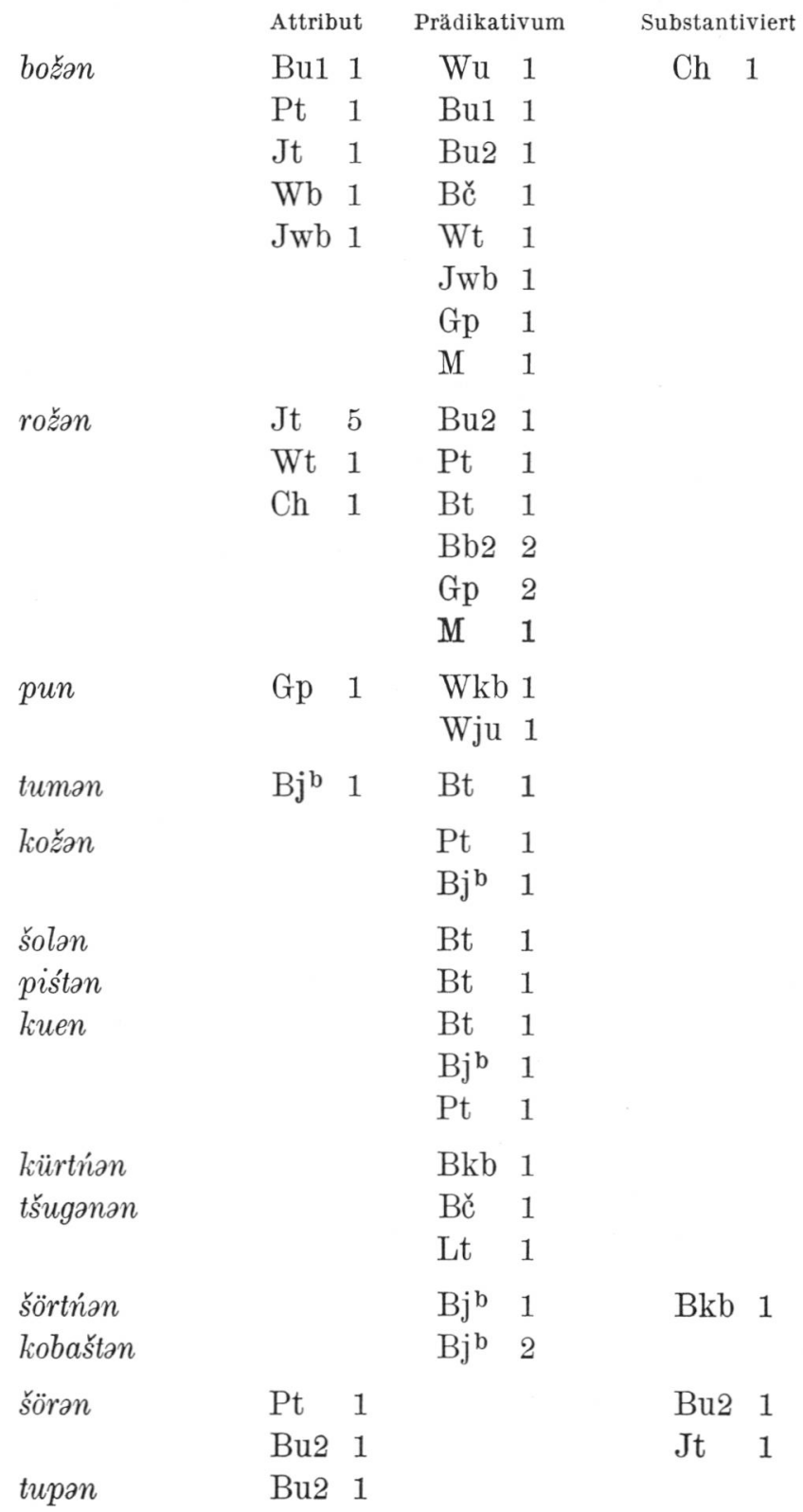


Es kommen nur die Genitivformen 14 verschiedener Wörter in Frage. Die Belegzahlen sind eigentlich zu hoch, denn z.B. die 5 Beispiele der Form rožan bei Jt stehen in den Varianten ein und desselben Rätsels. Von den attributivischen Belegen gehen 12 bzw. an $70 \%$ auf das Konto der Formen božan oder rožən, von den prädikativischen 16 bzw. mehr als $50 \%$. Ein gewisser Systemzwang bzw. der Einfluss eines in der Nähe stehenden possessiven Genitivs wird z.B. in der von Genetz aufgezeichneten Strophe sichtbar: ki əəštem ulo šište kudər korka, Onatər puən korkaže 'in meiner Hand ist ein maserhölzerner Becher, ein Onatər-hölzerner Becher', denn das Gedicht geht weiter: nemnan onžəmo oš ku $\gamma^{\prime}$ ža Onatər puən śaśkaže 'der von uns verehrte weisse Zar ist die Blüte des Onatər-Baumes' Gp 74. Eine Art Wortformenspiel wird in dem Rätsel sichtbar: Saßeli apšatan, Apati pun 'Sabellius gehört dem Schmied, Apati ist aus Holz' Wkb 135.

Die Genitivformen begegnen in diesen Beispielen in Stellungen, die nach der oben angedeuteten ergänzenden syntaktischen Distribution ein Possessivadjektiv voraussetzten. ANAbleitungen kommen denn auch von denselben Wörtern vor: ik munDəram šəm rožan 'mein Knäuel hat sieben Löcher' Wt

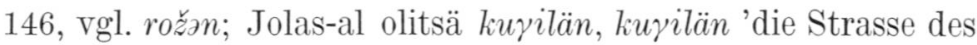
Dorfes Jolas-al ist mit Birken, Birken bewachsen' Wkb 180, vgl. kuen. Das Wörterbuch von Serebrennikov kennt die Formen rožan, vožzn und pun, neben denen rožan, vožan und sogar vožznan angeführt werden; AN-Ableitungen werden in dem Wörterbuch i.a. nicht aufgezählt. Das Stammwort vož bezeichnet sowohl 'Wurzel' als auch 'Zweig', die Ableitungen sind dann bedeutungsmässig differenziert: vožan 'wurzelig', vožan und weiter davon vožanan 'verzweigt'. Das im Genitiv stehende rožan bedeutet dagegen ungefähr dasselbe wie die Adjektivableitung rožan: ersteres ist 'zerrissen, löcherig', letzteres dagegen nur 'löcherig' übersetzt. In einigen Paaren ist der Bedeutungsunterschied übrigens klar: šörtrian 'golden' bedeutet etwas anderes als šörtrian 'reich an Gold'. Leider liefern die Textquellen sehr wenig Beispiele, doch gibt uns die Ableitung šian von einem anderen Metallnamen einen Hinweis

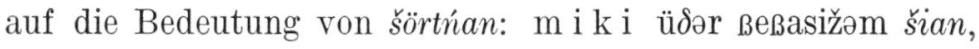


šian manət ələ 'Michaels Tochter Feodosia hatten sie als reich an Silber, an Silber bezeichnet' (nicht etwa als 'silbrig') Jt. Entsprechend bedeutet z.B. kürtnan 'reich an Eisen', was nicht dasselbe ist wie 'eisern' usw. Die genannten Genitivformen sind zumindest bedeutungsmässig keine possessiven Adjektive. Das ist auch gar nicht befremdlich, denn alle 14 Genitive vertreten Wörter, von denen ein possessiver, also ein adnominaler Genitiv normalerweise überhaupt nicht verwendet wird. Als adnominale Bestimmungen stehen diese Wörter im Nominativ.

Für die syntaktischen Beziehungen der oben aufgezählten Genitivformen sind jene Beispiele aufschlussreich, wo das als Attribut oder Prädikativum stehende A d j e kt i v ein Genitivsuffix erhalten hat.

A. Adjektiv $+\mathrm{N}$ als Attribut.

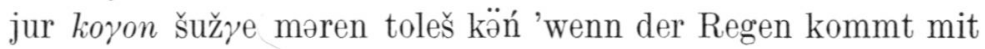
grossem Rauschen' Bkb 27.

kükśan kükśən kurəket Balnə jolan kilat šińd'ž́a 'auf einem

hohen, hohen Berg steht ein Säulenspeicher' Bu2 358. püa püaš moštədəmə kukšən koremeš püaleš 'wer kein Wehr bauen kann, baut es in eine trockene Schlucht' Bu2 504. tsijen olot sajən Bor reməm 'sie hatten sich schöne Kleider angezogen' $\mathrm{Bj}^{\mathrm{b}} 330$.

Auch das meist adjektivisch auftretende šukə 'mancher, viel' begegnet entsprechend in der Genitivform:

šukan erye artanam rualeš 'Gebrüder [eig. viele Söhne] hauen Holz' Wb 393.

In einem Beispiel von Jewskij steht das Verbalnomen - ̌̌e, das allgemein als Adjektivadjunkt in der Grundform vorkommt, im Genitiv:

tə tüńčašte memnan gańe lütšan jalnək uke 'in dieser Welt gibt es keine uns ähnlichen furchtsamen Tiere (keine so furchtsamen Tiere wie wir es sind)'.

B. Adjektiv $+\mathrm{N}$ als Prädikativum

šijžat šörtńəžat šergan ogəl, šočmaš kušmaš məlandəže šergə

'weder Silber noch Gold sind teuer, das Geburts- und das "Wachs»land (= die Heimat) ist teuer' Lt 49.

Dies ist denn auch das einzige deutliche Beispiel für das Auftreten des adjektivischen Genitivs in prädikativem Verhältnis 
- ein Beweis dafür ist die Verneinungsform des Verbs, ogəl.

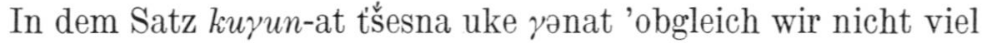
anzubieten haben' Wu 302, kann kuүun kein Prädikativum sein, da sich uke als Verneinungsform auf lokale und possessive Beziehungen beschränkt.

C. Adjektiv $+\mathrm{N}$ substantiviert.

pešk̇ðənam pi ok kotšs 'der Hund frisst kein hartgewordenes

(Fressen)' Jt F I 269.

Die Genitivform des Adjektivs begegnet als Attribut insgesamt 44mal (die Genitivform des Substantivs nur 17mal), als Prädikativum und substantiviert je einmal. Die Beispiele der erstgenannten Gruppe stammen grösstenteils (34) aus in gebundener Rede abgefassten Teilen der Textquelle Bu2. In einem dieser Dialekte sind alle Adjektive durchweg N-förmig, sobald sie in Gedichten auftreten, was natürlich ihre Beweiskraft schwächt. Einige als Attribut auftretende Adjektiv-

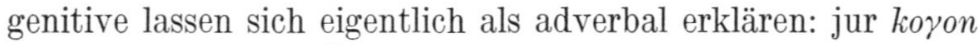
šužje məren toleš kə̈ń 'wenn der Regen kommt mit grossem Rauschen' liesse sich vielleicht auch analysieren als 'wenn der Regen kommt gross rauschend'. Das wiederholt vorkommende kükśan kükśan kurəket ßalnə wird in der Quelle Bu2 übersetzt 'am hohen Berggipfel', in der Textsammlung von Porkka dagegen lautet die Stelle 'hoch, hoch auf dem Berge', als adverbaler Genitiv aufgefasst also. Im Wörterbuch von Serebrennikov gibt das Stichwort kükšən-kükšan die Bedeutung 'hoch oben' (высоко-высоко). Das als Attribut vorkommende šukan ist recht interessant. Es könnte ein adnominaler, substantivischer Genitiv sein: šukan šərəm pi ak katšs 'der Hund frisst nicht merdam multorum' Bkb 14. In Wichmanns Sammlung bedeutet das šukan erye in einer Strophe der Texte aus Birsk jedoch kein possessives Verhältnis: in der Übersetzung 'viele Söhne'. Vgl. im gleichen Gedicht den entgegengesetzten Ausdruck əšket er $\gamma$ e 'der einzige Sohn', wo das i.a. als Adverb begegnende šket 'allein' die Stellung eines Attributs einnimmt. So lässt sich hier auch die Form šukan als Kasusformattribut ansehen: der normalerweise adverbal begegnende Ausdruck 'viel' ist in eine adnominale Stellung gelangt. Ebenso erklären sich kukšan koremeš 'in eine trocken seiende Schlucht', sajan 
Buryeməm 'die in gutem Zustand befindlichen Kleider', und auch lütšan jalnək 'die in Furcht seienden Tiere'. Die Adjektive sind Wörter, die nie als adnominale Genitive auftreten, unbegrenzt dagegen als adverbale Genitive. So lassen sich die als Attribute vorkommenden Genitivformen von Adjektiven erklären als adnominale Vorkommen einer i.a. adverbalen Kasusform, als Kasusformattribute oder ebenso gut als Adverbattribute.

Die syntaktische Distribution der adjektivischen Genitivformen gibt auch für die Auslegung der entsprechenden substantivischen Genitive Hinweise. In einigen Fällen könnte die als Attribut zu deutende Form mit dem Verb in Zusammenhang gebracht werden: pašu pokšelnə šörən kußa košteš 'mitten auf der Wiese geht eine Frau mit krummem Rücken, krummrückig', vgl. šəmašßlakat šöran kijat ulmaš 'die Steinbeeren lagen auf ihrer Seite' Pab 7. Einige Formen sind eindeutig Kasusformattribute adverbieller Art: оßба tupan jolan 'Owda hat verkehrte Füsse'. In den meisten Dialekten erscheint denn auch in der entsprechenden Bedeutung das auf -n ausgehende Adverb tupəń. Der gleiche Typus steht als Adverbattribut z.B. in dem Satz koton šomažəm jenlan pua marelan 'den senkrechten Mund gibt sie einem anderen' $\mathrm{Bj}^{\mathrm{a}}$ 85. Ein völlig adverbieller Genitiv eines Verbalstamms begegnet in attributiver Stellung in dem Ausdruck šočən aba 'Geburt-Mutter'. E. Itkonen hat ja kürzlich (Virittäjä 1/1966) darauf hingewiesen, dass in vielen finnisch-ugrischen Sprachen das Adverb als Bestimmung des Nomens relativ allgemein vorkommt.

Das aus einem adverbalen Verhältnis abstrahierte Kasusformattribut ist im Tscheremissischen auch sonst bekannt. Hier kommt besonders eine Umstandsbestimmung des Ortes oder eine Postpositionskonstruktion in Frage:

maska užən marenəm komakamßalne pərəsəm 'der Bär erblickte des Mannes oben auf dem Ofen seiende Katze' Bjb 272. Das an den Genitiv tretende Akkusativsuffix maren-əm ist folgendermassen zu analysieren: der Genitiv tritt an die Form pərəsəm; nicht also 'der Bär sah auf dem Ofen des Mannes eine Katze'. 


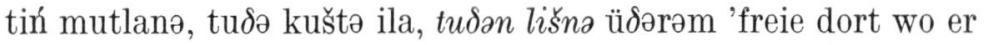
wohnt, ein in seiner Nähe wohnendes Mädchen' Bu1 156.

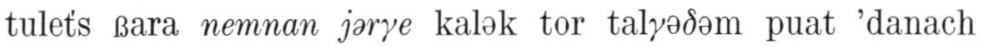
opfert das um uns herum wohnende Volk ein braunes Fohlen' Bb1 62.

nənən baštek läktät paštek marəßə̈lä 'nach ihnen gehen die Begleitmänner' Bkb 72.

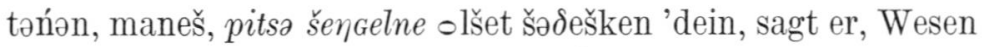
hinter dem Zaun wurde böse' Bjb 129. - Hier zeigt das Personalzeichen, dass tańan olšet zusammengehört, nicht also 'hinter deinem Zaun'.

Aufgrund des oben Dargestellten lassen sich auch die Genitivformen von Substantiven, die als Adjektivattribute stehen, als Kasusformattribute erklären. Die Genitivformen eines Substantivs in der Stellung des Prädikativums können auf der gleichen Basis ausgelegt werden: als Kasusformen und nicht als Ableitungen. Man kann sie als adverbale Genitive betrachten, auch wenn der Satz kein Prädikatsverb enthielte: Ajək pondaš ßožan 'Ajəks Bart ist gespalten, verzweigt' Wt 139. Vgl. das Auftreten des Genitivs eines Verbalstamms in einem entsprechenden Ausdruck: tšeßer jəฤgajən müškürržö šelan 'der Bauch meiner schönen Schwägerin ist geplatzt' Jt, F I. Bereits Gauthiot wies seinerzeit darauf hin, dass in den wolgafinnischen Sprachen, im Mordwinischen und Tscheremissischen also, die erwartungsgemässe Form vom Verb s e i n zur Vertretung der 3. Sg. Prs. Ind. nicht nur in Prädikativsätzen fehlen kann, sondern speziell auch in solchen, in denen eine Ortsbestimmung enthalten ist: tińən kušto ćonet? 'wo hast du deine Seele?' Gp 14. Die Feststellung lässt sich noch verallgemeinern: die Form vom Verb 'sein', die die dritte Person Singularis Präsentis im Indikativ vertritt, erscheint in einem Satz mit einem Prädikativverhältnis überhaupt nicht, kann aber auch in einem anderen Ausdruck mit jedem beliebigen Verhältnis fehlen. So fehlt sie im possessiven Verhältnis im folgenden Satz: ši $\gamma$ ukužən kəm i $\gamma \ddot{\partial}$, mänmän äßän kut i $\gamma \ddot{\partial}$ 'der Silberkuckuck (hat) drei Junge, unsere Mutter sechs Kinder' Bkb 351. In einem Satz mit einem adverbalen Genitiv fehlt die entsprechende finite Verbform z.B. hier: Patambal arka kečəlan melan 'der oberhalb 
des Patam-Stromes liegende Abhang liegt gegen die Sonne (eig. der Sonne gegenüber)' Gp 73. Das fehlende Prädikatsverb z.B. in dem Ausdruck Ajək pondaš $\beta$ ožzn beweist also nicht" unbedingt, dass $\beta_{0}$ žn hier als Prädikativum fungiert, in der Funktion der Nominativform des Adjektivs - es kann ebenso gut in der Funktion der Genitivform des Adjektivs auftreten. Zu beachten ist, dass sich für den adverbalen Genitiv unmöglich eine genaue Bedeutungsaufgabe nachweisen lässt: seine jeweilige Bedeutung hängt in erster Linie von der Satzstruktur ab, dann von der Bedeutung des Verbs, dann von der eigenen lexikalischen Bedeutung des Wortes und schliesslich vom Sachzusammenhang. Dass z.B. in dem Satz kalak pun (Jewskij) 'der Löffel ist aus Holz' pun wie ein Prädikativum »wirkt», hängt davon ab, dass das Wort die Materie bezeichnet. Wenn der Satz kalak šöran lautete, würde der Genitiv ohne weiteres adverbal aufgefasst: 'der Löffel liegt auf der Seite'.

Von deskriptiver Seite liegt kein Hindernis vor, das Verhältnis kalak pun als prädikativ zu erklären. Dann muss man nur für das tscheremissische Prädikativum zwei Kategorien annehmen: eine totale und eine partielle. Zwischen Subjekt und totalem Prädikativum herrscht ein begriffliches Gleichmass: der Bereich des Prädikativums entspricht dem des Subjekts. Der Bereich des partiellen Prädikativums wird dagegen nicht durch das Subjekt gedeckt. Der Kasus des totalen Prädikativums ist der Nominativ, der des partiellen der Genitiv. Die Genitivform ist nur als Adjektivableitung zu erklären, falls sie als totales Prädikativum erscheint.

Somit sind die Fälle, wo die Genitivform in der syntaktischen Funktion des Adjektivs erscheint, aufgrund des adverbalen, nicht des adnominalen Genitivs zu erklären. Hierauf weist auch die Seltenheit der Belege hin. Die adverbale Form kann zwar an der Stelle einer attributiven Form in Frage kommen, doch handelt es sich dann im Hinblick auf die syntaktische Gesamtstruktur der Sprache um eine Ausnahme. Andererseits kann auch eine attributive Form die Stellung einer erwarteten adverbalen Form einnehmen. Wie zu Beginn erwähnt gibt es kein einziges Beispiel, wo eine AN-Ableitung in der Genitivform aufträte. Im folgenden Ausdruck steht der AN-Typus im 
Nominativ, obgleich die sonstigen Adjektive den Genitiv zeigen: kölan kitšəlan šokšan tuťššs, kölan d'ükštan tuț̌seš, kölan punan tut'šeš 'wem es an der Hand heiss scheint, wem kalt, wem haarig' Bu2 110. Das Verb tut'sam verlangt regelmässig ein im Genitiv stehendes prädikatives Adverbiale. Die erwartungsgemässe Form lautet jedoch durchaus nicht punanan sondern punan. Von AN-Ableitungen braucht man keine Genitivform, da für die adverbale Funktion dieser Adjektive ohnehin eine syntaktische Kategorie vorliegt: die Genitivform des Stammworts.

Im heutigen Tscheremissisch sind der Genitiv - sowohl der adnominale als auch der adverbale - und das Possessivadjektiv deutlich voneinander unterschiedene syntaktische Kategorien, obgleich sie in Ausnahmefällen mitunter ihre Funktionen auszutauschen scheinen. Historisch hängen diese Formen sicher miteinander zusammen; beide besitzen ja ein gemeinsames N-Element, wenn auch beim Possessivadjektiv der Bindevokal zum Träger der Bedeutungsfunktion wurde. Unter rein lautlichen Gesichtspunkten können die in adjektivischer Funktion begegnenden Genitivformen eines Substantivs als alte Relikte gelten; die syntaktischen Gesichtspunkte weisen dagegen auf Beziehungen hin, die sich mittels der modernen Sprache erklären lassen.

Eeva KangasmaA-Minn 


\section{Abkürzungen der Textquellen}

Wkb = Wichmann, Yrjö, Volksdichtung und Volksbräuche der Tscheremissen. MSFOu 59, (1931) S. 1-10, 113-114, 133-136, $170-234$.

$\mathrm{Bkb}=$ Beke, Ödön, A cseremiszek (marik) népköltészete és szokásai 1. Budapest 1951.

$\mathrm{Bj}^{\mathrm{a}}=$ Beke, Ödön, Tscheremissische Texte zur Religion und Volkskunde. Oslo Etnografiske Museum, Bulletin 4. Oslo 1931.

$\mathrm{Bj}^{\mathrm{b}}=$ Beke, Ödön, Mari szövegek, I kötet, S. 6-351. Budapest 1957.

Bjc = Beke, Ödön, Tscheremissische Märchen aus dem Kreise Jaransk. Opetatud Eesti Seltsi Aastaraamat 2. Tartu 1937.

$\mathrm{Wju}=$ Wichmann, a.a.O., S. 74-112, 115-124, 136-139, 245-254.

$\mathrm{Wu}=$ Wichmann, a.a.O., S. $12-17,27-58,124-127,139-145$, 156-163, 254-306.

Bu1 = Beke, Ödön, Tscheremissische Märchen, Sagen und Erzählungen, MSFOu 76 (1938).

Bu2 = Beke, Ödön, Mari szövegek, III kötet. Budapest 1961.

$\mathrm{Brc}=$ Beke, Ödön, Mari szövegek, I kötet, S. 556-679. Budapest 1957.

Pt = Porkka, V. (Genetz), Volmari Porkkas tscheremissische Texte mit Übersetzung. JSFOu $13 \mid 1$ (1895).

$\mathrm{Wt}=$ Wichmann, a.a.O., S. $17-21,58-66,127-130,145-153$, $164-168,306-364$.

Lt = Lewy, Ernst, Tscheremissische Texte I. Hannover 1926.

Bt = Beke, Ödön, Mari szövegek, I kötet, S. 364-552. Budapest 1957.

$\mathrm{Jt}=$ Jevsevjev collection.

$\mathrm{Bm}=$ Beke, Ödön, Mari szövegek, IV kötet, S. 10-145. Budapest 1961.

$\mathrm{Wb}=$ Wichmann, a.a.O., S. $385-476$ (Von Karmazin gesammelte Lieder).

$\mathrm{Pab}=$ Paasonen, H., Tscheremissische Texte. Herausgegeben von Paavo Siro. MSFOu 78 (1939).

$\mathrm{Bb1}=$ Beke, Ödön, Texte zur Religion der Osttscheremissen. Anthropos 29 , S. $39-69,371-398,703-737$ (1939). 
Bb2 = Beke, Ödön, Mari szövegek, IV kötet, S. 153-447. Budapest 1961.

$\mathrm{Jwb}=$ Von Iwan Jewskij erhaltenes Material.

Gp = Genetz, Arvid, Ost-Tscheremissische Sprachstudien I. JSFOu 7 (1889).

I = Iljakov, N., Edemvlä dä ivlä. Kozmodemjansk 1957.

Ch = Chavain, S.G., Elnet. Joškar-Ola 1958.

M = Mikhailov, Alexander, Korno vožešto. Joškar-Ola 1959. 